OPEN ACCESS

Edited by:

Braxton A. Norwood,

Expesicor LLC, United States

Reviewed by:

Dinesh Upadhya,

Manipal Academy of

Higher Education, India

Chinmaya Sadangi,

University of Toronto, Canada

*Correspondence:

Zucai Xu

docxzc@126.com

tThese authors have contributed equally to this work

Specialty section:

This article was submitted to

Epilepsy,

a section of the journal

Frontiers in Neurology

Received: 08 April 2018

Accepted: 11 October 2018

Published: 29 October 2018

Citation:

Yang Z, Wang J, Yu C, Xu P, Zhang J,

Peng $Y$, Luo Z, Huang $H$, Zeng J and

Xu Z (2018) Inhibition of p38 MAPK Signaling Regulates the Expression of EAAT2 in the Brains of Epileptic Rats.

Front. Neurol. 9:925

doi: 10.3389/fneur.2018.00925

\section{Inhibition of p38 MAPK Signaling Regulates the Expression of EAAT2 in the Brains of Epileptic Rats}

\author{
Zhang Yang ${ }^{1,2+}$, Jing Wang ${ }^{3 \dagger}$, Changyin $\mathrm{Yu}^{1}$, Ping $X u^{1}$, Jun Zhang ${ }^{1}$, Yan Peng ${ }^{1}$, \\ Zhong Luo ${ }^{1}$, Hao Huang ${ }^{1}$, Junwei Zeng ${ }^{4}$ and Zucai Xu ${ }^{1,5 *}$
}

${ }^{1}$ Department of Neurology, Affiliated Hospital of Zunyi Medical University, Zunyi, China, ${ }^{2}$ Department of Neurology, Affiliated Hospital of Guizhou Medical University, Guiyang, China, ${ }^{3}$ Department of Prevention and Health Care, Affiliated Hospital of Zunyi Medical University, Zunyi, China, ${ }^{4}$ Department of Physiology, Zunyi Medical University, Zunyi, China, ${ }^{5}$ Key Laboratory of Brain Science, Zunyi Medical University, Zunyi, China

Seizures induce the release of excitatory amino acids (EAAs) from the intracellular fluid to the extracellular fluid, and the released EAAs primarily comprise glutamic acid (Glu) and asparaginic acid (Asp). Glu neurotransmission functions via EAA transporters (EAATs) to maintain low concentrations of Glu in the extracellular space and avoid excitotoxicity. EAAT2, the most abundant Glu transporter subtype in the central nervous system (CNS), plays a key role in the regulation of glutamate transmission. Previous studies have shown that SB203580 promotes EAAT2 expression by inhibiting the p38 mitogen-activated protein kinase (MAPK) signaling pathway, but whether SB203580 upregulates EAAT2 expression in epileptic rats is unknown. This study demonstrated that EAAT2 expression was increased in the brain tissue of epileptic rats. Intraperitoneal injection of a specific inhibitor of p38 MAPK, SB203580, reduced the time to the first epileptic seizure and attenuated the seizure severity. In addition, SB203580 treatment increased the EAAT2 expression levels in the brain tissue of epileptic rats. These results suggest that SB203580 could regulate epileptic seizures via EAAT2.

Keywords: epilepsy, glutamic acid, SB203580, excitatory amino acids, p38 mitogen-activated protein kinase

\section{INTRODUCTION}

Epilepsy is a global public health problem. In fact, there are $\sim 70$ million epilepsy patients worldwide (1), and more than $90 \%$ of these patients are located in low- and middle-income countries. According to statistical data, epilepsy accounts for $0.7 \%$ of the global burden of disease. Repeated epileptic seizures have negative effects on mood, behavior, personality, cognition, movement, and sleep and can even increase mortality in patients with epilepsy (2).

Imbalances in the levels of central nervous system (CNS) neurotransmitters are important mechanisms in epilepsy. Epileptic seizures can increase the release of excitatory amino acids (EAAs), which primarily include glutamic acid (Glu) and asparaginic acid (Asp), from inside the cell to the outer environment (3). Glu is the major excitatory neurotransmitter of the CNS, and abnormal expression levels of its receptor and transporter are closely related to the pathogenesis of epilepsy (4). Studies have shown that seizures in epileptic patients can abnormally increase the extracellular Glu and Glu receptor levels, and these increased levels of EAAs increase the number of CNS neurons in a state of excessive excitability (5). Through brain microdialysis, other scholars have shown that extracellular Glu levels continue to increase before and during seizures in epilepsy 
patients $(3,6)$; in addition, as demonstrated in a rat model of epilepsy, excessive increases in the extracellular Glu levels lead to toxic neuronal excitability and might eventually lead to neuronal death after seizures $(7,8)$. EAA transporter 2 (EAAT2) is distributed in the hippocampus and nerve cells of the prefrontal lobe in mammals. This transporter can reduce nerve cell injury by absorbing extracellular Glu and transporting it into cells, and this process reduces the Glu levels in the synaptic cleft and decreases the excitotoxicity of Glu to neurons and glial cells (9-12). In addition, there is a type of stress-activated protein kinase in the mammalian CNS through which mitogen-activated protein kinase (MAPK) can transmit extracellular signals to the nucleus (13). This kinase, p38 MAPK, is a member of the MAPK family, which plays an important role in cell survival, differentiation and development. However, activation of p38 MAPK in the CNS causes neuronal cell death (14). Studies have shown that in the CNS, the binding of Glu to the NMDA receptor can activate p38 MAPK and induce the apoptosis of hippocampal granule cells in the brain (15). In addition, Okamoto performed a genomewide screen to show that p38 MAPK is overexpressed in the hippocampus of epileptic rats (16). Consequently, p38 MAPK might play a role in epilepsy. Additionally, a study by Liang et al. showed that activated $\delta$ opioid receptors potentially regulate the methyl ethyl ketone (MEK)-extracellular signal-regulated kinase (ERK)-p38 MAPK cascade transduction pathway to promote the expression of EAAT1 and EAAT2 in astrocytes and decrease the concentration of extracellular Glu (17). In summary, p38 MAPK, Glu and EAAT2 appear to be associated with epilepsy, and SB203580 competitively binds to ATP binding sites for the purpose of inhibiting p38MAPK, thereby effectively inhibiting some signal transduction induced by some inflammatory factors. However, whether the regulation of p38 MAPK is related to the expression of EAAT2 during epileptic seizures has not been reported in the literature. Therefore, this study assessed the changes in the epileptic seizure intensity and in the expression levels of EAAT2 in brain tissues induced by the p38 MAPK inhibitor SB203580 in a lithium chloride-pilocarpine-induced rat model of epilepsy.

\section{MATERIALS AND METHODS}

\section{Establishment of the Lithium Chloride-Pilocarpine-Induced Rat Model of Epilepsy}

Male Sprague Dawley (SD) rats weighing $\sim 180-220$ g were purchased from the Experimental Animal Center of the Third Military Medical University [clean facility; license number: SCXK, (Yu) 2012-0005]. All animal experiments were performed in accordance with the Guidelines for Animal Experiments of the Chinese Academy of Medical Sciences and with approval from the Ethics Committee for Animal Care of the Third Military Medical University. The rats were maintained in an environment with a constant temperature $\left(24 \pm 2{ }^{\circ} \mathrm{C}\right)$ and received pure drinking water and standard feed via conventional cage feeding. A total of 162 adult male SD rats were included in this study. Forty-seven were excluded ( 32 rats died and 15 failed). The rats were randomly divided into the following groups: the untreated control, the epilepsy group $(0 \mathrm{~h}, 1 \mathrm{~d}, 3 \mathrm{~d}, 1 \mathrm{w}$, and $2 \mathrm{w})$, the SB203580 group, and the solvent control group (normal saline $+2 \%$ DMSO).

Randomly selected healthy adult male $\mathrm{SD}$ rats received an intraperitoneal injection of lithium chloride $(127 \mathrm{mg} / \mathrm{kg}) ; 20 \mathrm{~h}$ later, the rats were intraperitoneally injected with atropine sulfate $(1 \mathrm{mg} / \mathrm{kg}$ ), and $30 \mathrm{~min}$ later, the rats were intraperitoneally injected with pilocarpine $(50 \mathrm{mg} / \mathrm{kg})$. Seizure behaviors after kindling were classified according to the standards of the Racine scale as follows: stage I, mouth and facial movements; stage II, head nodding; stage III, forelimb clonus; stage IV, rearing and stage $\mathrm{V}$, rearing and falling. If the rats did not have a Racine score of IV-V after $30 \mathrm{~min}$ (18), single intraperitoneal injections of pilocarpine $(10 \mathrm{mg} / \mathrm{kg})$ were administered until seizure development. After the rats achieved status epilepticus (SE) and maintained this status for $45 \mathrm{~min}$, they were administered an intraperitoneal injection of atropine sulfate $(1 \mathrm{mg} / \mathrm{kg})$ and diazepam $(10 \mathrm{mg} / \mathrm{kg})$ to terminate the seizure. The behaviors of the rats were monitored and recorded, and the Racine scores of the experimental group reached IV-V. The rats in the untreated control group received the same treatment with lithium chloride and atropine sulfate, but were administered saline instead of pilocarpine. The rats in the SB203580 group received an intraperitoneal injection of SB203580 (15 mg/kg; normal saline $+2 \%$ freshly prepared DMSO) (19) 30 min before the pilocarpine injections, and the rats in the solvent control group received the same concentration of DMSO as the SB203580 group.

\section{Western Blotting}

To collect brain tissue from each group of rats, the rats were anesthetized with $10 \%$ chloral hydrate $(0.33 \mathrm{ml} / 100 \mathrm{~g})$ by intraperitoneal injection. After administration of the anesthesia, the skull was rapidly removed and separated, and the brain tissue was removed. The bilateral hippocampus and the cortex of the adjacent temporal lobe were quickly separated from the bath, and the specimen was wrapped with tin foil, placed in a cryotube and stored in a liquid nitrogen tank. All the samples were homogenized in $30 \mathrm{mM}$ Tris- $\mathrm{HCl}$ and $100 \mathrm{mM}$ phenylmethylsulfonyl fluoride. The protein concentrations of the supernatants were measured using an Enhanced BCA Protein Assay Kit (Beyotime, Haimen, China) according to the manufacturer's instructions. Equal amounts of protein samples were run on SDS-PAGE gels (5\% stacking gel; $10 \%$ separation gel) and transferred to polyvinylidene difluoride membranes. The PVDF membranes had been pretreated with methanol for $\sim 5 \mathrm{~min}$, and the filter paper and sponge were soaked into the electro-transfer buffer during storage. The gel was sandwiched between the sponges and filter paper and rolled with a roller to remove bubbles. The sandwich was electro-transferred for $4 \mathrm{~h}$ at $40 \mathrm{~V}$, and the membrane was blocked in 5\% skim milk at RT for 3-4 h. After this process, the PVDF membrane was removed and incubated overnight at $4^{\circ} \mathrm{C}$ with anti-EAAT2 (rabbit monoclonal antibody, 1:2,000 dilution, Abcam, ab203130) and then at $23^{\circ} \mathrm{C}$ for $2 \mathrm{~h}$ with HRP-tagged secondary antibody $(1: 1,000$, Santa Cruz Biotechnology, CA, USA, sc-2004). Blots were 
developed using Super Signal West Pico Chemiluminescent HRP substrate (Rockford, IL, USA) according to the manufacturer's instructions, and the blot intensities were calculated using Quantity One software (Bio-Rad Laboratories, Hercules, CA, USA).

\section{Immunohistochemistry Staining}

The anesthetized animals were transcardially perfused with saline and then with $4 \%$ formaldehyde in phosphate buffer $(\mathrm{pH} 7.4)$ for $10 \mathrm{~min}$. The brains were then carefully removed, post-fixed at $4^{\circ} \mathrm{C}$ in the formaldehyde solution for $24 \mathrm{~h}$, dehydrated in a series of graded ethanol solutions and cleared in xylene. The samples were subsequently embedded in paraffin, and 4 - to 5 - $\mu$ m-thick coronal sections were cut.Paraffin sections were deparaffinized in xylene and rehydrated in a graded series of ethanol solutions. After three 15-min washes with phosphate-buffered saline, the sections were incubated in $3 \% \mathrm{H}_{2} \mathrm{O}_{2}$ for $15 \mathrm{~min}$, heated $\left(92-98^{\circ} \mathrm{C}\right)$ in $10 \mathrm{mmol} / \mathrm{l}$ boiling sodium citrate buffer $(\mathrm{pH} \mathrm{6.0)}$ for $20 \mathrm{~min}$ and blocked with normal goat serum (1:10) for $10 \mathrm{~min}$ (Gene Tech Inc., Shanghai, China).The sections were then incubated with anti-EAAT2 (rabbit monoclonal antibody, 1:200 dilution, Abcam, ab203130) at $37^{\circ} \mathrm{C}$ for $2 \mathrm{~h}$, subjected to three 30 -min washes with PBS, incubated with biotinylated goat anti-rabbit secondary antibody (Gene Tech Inc., Shanghai, China, abs20003) for $30 \mathrm{~min}$ and washes again. The sections were subsequently treated with $\mathrm{ABC}$ solution for $30 \mathrm{~min}$ at $37^{\circ} \mathrm{C}$ and washed three times with $\mathrm{PBS}$ ( $5 \mathrm{~min}$ each time). DAB coloring solution was then dropped onto the sections. As determined by observation using a microscope, when brownish yellow particles appeared, pure water was added to terminate the coloration reaction. The paraffin sections were rehydrated in a graded series of ethanol solutions and deparaffinized in xylene. An Olympus PM20 automatic microscope (Olympus, Osaka, Japan) and a TCFY-2050 pathology system (Yuancheng Inc., Beijing, China) were used for image collection. If the cytoplasm was dyed a yellowish color, the sample was considered positive for EAAT2. For each sample, images of ten random visual fields at 20x magnification were selected for analysis. The HPIAS 1000 high-definition image analysis system (Qianping Image Technology Co., Ltd., Wuhan, China) was used to count the EAAT2-positive cells.

\section{Immunofluorescence Staining}

To confirm the location of EAAT2 in the neurons and astrocytes in the hippocampus after seizure, the immunoreactivity for EAAT2 in rats $3 \mathrm{~d}$ after seizure onset was observed by double-labeling immunofluorescence. Tissues sections were permeabilized with $0.5 \%$ Triton X-100 and incubated first with $10 \%$ goat serum (Zhongshan Golden Bridge, Inc., Beijing, China) for $30 \mathrm{~min}$ and then with rabbit anti-EAAT2 (1:200; Abcam, Cambridge, UK, ab203130) and mouse anti-GFAP antibodies (1:100; Santa Cruz Biotechnology, USA, sc-71143) overnight at $4^{\circ} \mathrm{C}$. The next day, the sections were washed five times with phosphate-buffered saline (55 min total) and incubated in the dark with secondary antibodies (anti-rabbit-Alexa 405, green, Abcam, Cambridge, UK, ab169345; anti-rabbit-Alexa 488, blue, Boster, Inc., Wuhan, China, F06630-1; anti-rabbit-Alexa 549, red, Zhongshan Golden Bridge, Inc., Beijing, China, SC-549) at 1:100 dilution for $60 \mathrm{~min}$. The sections were then counter-stained with DAPI (4',6-diamidino-2-phenylindole, 1:10,000 dilution, SigmaAldrich, D9542) and PBS for 20 min, mounted and sealed with $50 \%$ glycerin, and the slides were then observed using a laser scanning confocal microscope at 40x magnification.

\section{Statistical Methods}

The data are expressed as the means \pm standard deviations and were analyzed using SPSS version 18.0. The behavioral characteristics of the rats (seizure duration and Racine score) and the immunohistochemistry and Western blot results were compared by two-way ANOVA; $P<0.05$ was used to indicate a significant difference.

\section{RESULTS}

\section{Effects of SB203580 on the Duration of the First Seizure and Racine Scores in Rats}

The Racine scores of the epilepsy, solvent control and SB203580 groups were $2.57 \pm 0.48,2.55 \pm 0.52$, and 0.66 \pm 0.48 , respectively, $10 \mathrm{~min}$ after the intraperitoneal injection of pilocarpine; $3.37 \pm 0.50,3.96 \pm 0.55$, and $1.75 \pm 0.43$, respectively, at $20 \mathrm{~min}$ after the intraperitoneal injection of pilocarpine; and $4.88 \pm 0.46,4.87 \pm 0.45$, and $2.87 \pm 0.32$, respectively, at $30 \mathrm{~min}$ after the intraperitoneal injection of pilocarpine. The duration of the seizures in the epilepsy, solvent control and SB203580 groups was $62.56 \pm 9.22 \mathrm{~s}, 58.79 \pm 8.41 \mathrm{~s}$, and $28.35 \pm 4.00 \mathrm{~s}$, respectively. No significant differences in the Racine scores or seizure duration were found between the epilepsy and solvent control groups $(P>0.05)$. Compared with the epilepsy and solvent control groups, the Racine score in the SB203580 group was significantly decreased at the three tested time points after the pilocarpine injection, and the seizure duration was significantly shortened. The intraperitoneal injection of SB203580 significantly reduced the Racine scores and the duration of the first seizure in lithium chloride-pilocarpineinduced epileptic rats $(P<0.05)$; however, the effect of $2 \%$ DMSO on these parameters was not statistically significant (Figures 1, 2).

\section{Effects of Seizure and SB203580 (a Specific Inhibitor of p38 MAPK) on EAAT2 Expression in Brain Tissue}

A Western blot analysis was used to assess the expression levels of total and phosphorylated p38 protein (T-p38 and P-p38, respectively) in the untreated control, SB203580 and epilepsy groups. The immunoblot density ratios of T-P38 to the corresponding internal reference $(\beta$-actin) in the control, SB203580, and epilepsy groups were $0.195 \pm 0.012,0.174 \pm$ 0.015 , and $0.376 \pm 0.02$, respectively. The expression of T-P38 in the hippocampus of the epileptic rats was significantly increased $(P<0.05)$ compared with that in the untreated control and SB203580 rats. In addition, the immunoblot density ratios of P-p38 to the corresponding internal reference ( $\beta$-actin) in the control, SB203580, and epilepsy groups were $0.538 \pm 0.021,0.517$ \pm 0.018 , and $0.523 \pm 0.015$, respectively. No significant difference 


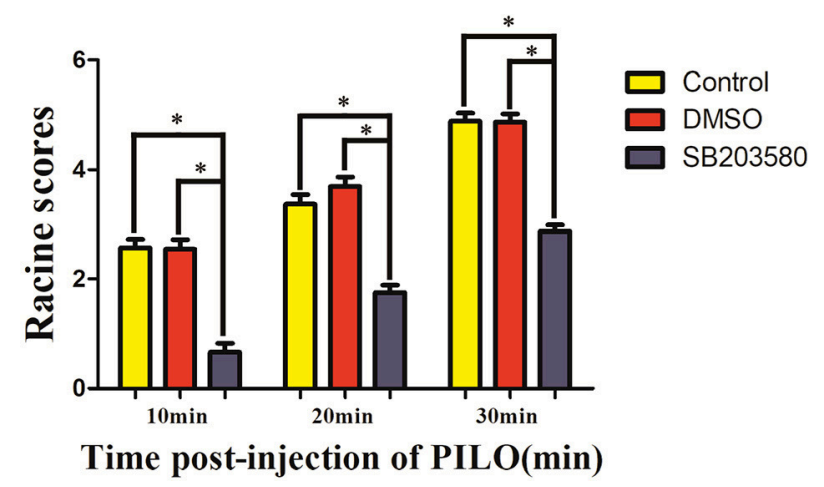

FIGURE 1 | Racine scores of different groups at different time points after the pilocarpine injection (min). ${ }^{\star} P<0.05$ compared with the control (epilepsy) and DMSO groups.

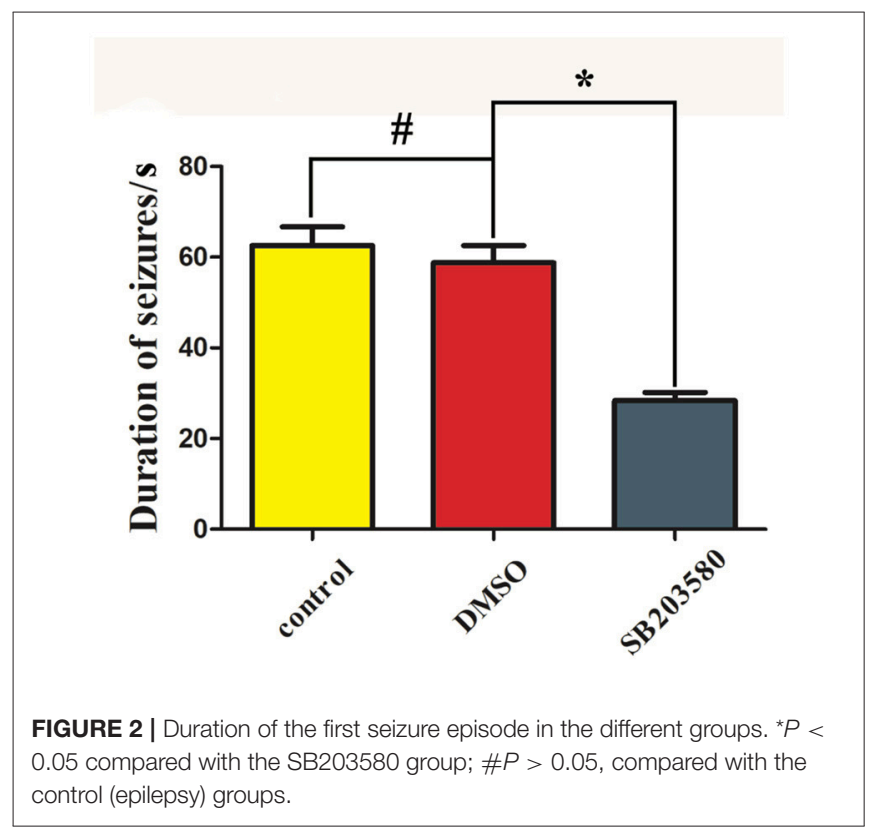

$(P>0.05)$ in $\mathrm{P}-\mathrm{p} 38$ expression was found between the untreated control group and the SB203580 group (Figure 3).

EAAT2 was expressed at basal levels in the hippocampus of the untreated control group, and the immunoblot density ratio of EAAT2 to the corresponding internal reference $(\beta$ actin) was $0.39 \pm 0.15$. In the epilepsy group, the immunoblot density ratios of EAAT2 to $\beta$-actin were $0.52 \pm 0.13,0.89 \pm$ $0.24,1.39 \pm 0.33,0.93 \pm 0.27$, and $0.43 \pm 0.16$ at different times. At the first three time points after the onset of seizure, the EAAT2 expression levels were significantly increased $(P$ $<0.05)$ compared with those of the untreated control group, and EAAT2 expression peaked at day 3. At 1 and $2 \mathrm{w}$ after seizure onset, the EAAT2 expression levels began to decrease to the normal levels. According to these results, the highest expression level of EAAT2 occurred $3 \mathrm{~d}$ after the onset of epileptic seizures. Therefore, $3 \mathrm{~d}$ after seizure onset was selected as the time point for measuring the effects of the p38 MAPK inhibitor SB203580. SB203580 was injected intraperitoneally $30 \mathrm{~min}$ before the seizure model was induced, and brain tissue proteins were extracted for Western blot analyses. In the rat hippocampus, the immunoblot density ratios of EAAT2 to $\beta$-actin in the SB203580 and solvent control groups were $2.19 \pm 0.46$ and $1.27 \pm 0.46$, respectively. The Western blot results showed that the expression level of EAAT2 in the SB203580 group at $3 \mathrm{~d}$ was significantly higher than that in the epilepsy group $(P<0.05)$. At $3 \mathrm{~d}$, no significant difference in EAAT2 expression was found between the solvent control group and the epilepsy group $(P>0.05)$ (Figure 4).

The Western blot results showed that EAAT2 was expressed at basal levels in the temporal neocortex of the rats in the untreated control group, and the immunoblot density ratio of EAAT2 to $\beta$-actin was $0.25 \pm 0.1$. In the epilepsy group, the immunoblot density ratios of EAAT2 to $\beta$-actin at various time points were $0.33 \pm 0.09,0.69 \pm 0.25,1.15 \pm 0.25,0.65 \pm 0.26$, and 0.28 \pm 0.09 . Specifically, From $0 \mathrm{~h}$ to $3 \mathrm{~d}$ after seizure onset, the EAAT2 expression levels were significantly increased $(P<0.05)$ in the epilepsy group compared with the untreated control group, and EAAT2 expression peaked $3 \mathrm{~d}$ after seizure onset. At 1 and $2 \mathrm{w}$ after seizure onset, the EAAT2 expression levels began to decrease to the normal levels. These results show that the highest expression level of EAAT2 occurred $3 \mathrm{~d}$ after the onset of epileptic seizures. Therefore, $3 \mathrm{~d}$ after seizure onset was selected as the time point for measuring the effects of the p38 MAPK inhibitor SB203580. SB203580 was injected intraperitoneally 30 min before the model was induced, and brain tissue proteins were extracted for Western blot analyses. In the temporal neocortex of rats, the immunoblot density ratios of EAAT2 to $\beta$-actin in the SB203580 and solvent control groups were 2.03 and 1.12, respectively. The Western blot results showed that EAAT2 expression in the SB203580 group at $3 \mathrm{~d}$ after seizure onset was significantly higher than that in the epilepsy group $(P<0.05)$, and no significant difference in EAAT2 expression was found between the solvent control group and the epilepsy group at this time point $(P>0.05)$ (Figure 5).

These results suggest that using SB203580 to inhibit p38 MAPK can increase the expression levels of EAAT2 in the hippocampus and neocortex of epileptic rats.

\section{Hippocampal EAAT2 Expression Measured by Immunohistochemistry and Effect of SB203580 on Its Expression}

The Western blot results showed that the protein expression levels of EAAT2 in the hippocampus of epileptic rats were significantly higher than those in the untreated control group, and the highest level of expression occurred $3 \mathrm{~d}$ after seizure onset. For this reason, $3 \mathrm{~d}$ after seizure was selected as the time point for measuring EAAT2 expression in the hippocampus by immunohistochemistry (Figure 6). The results showed that at $3 \mathrm{~d}$, EAAT2 was mainly expressed in the neuronal cell membrane of the CA1, CA3 and DG hippocampal areas in the untreated control, seizure and SB203580 groups. In addition, we performed a semi-quantitative analysis of the number of 


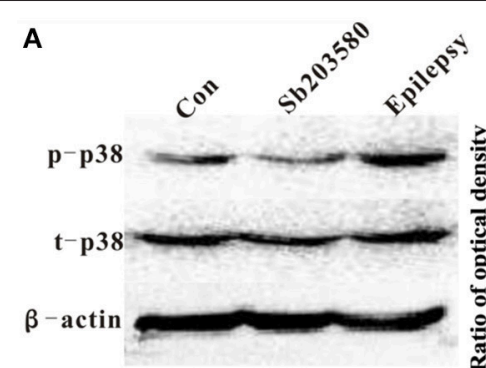

B
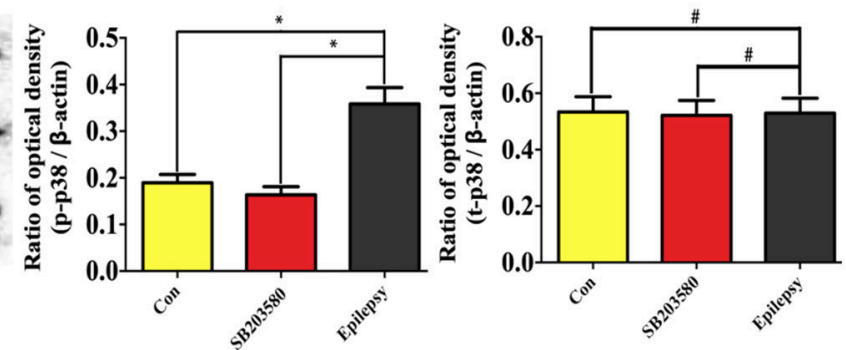

FIGURE 3 | Hippocampal t-p38 and p-p38 expression in the different groups measured by Western blotting. Panel (A) shows the Western blotting image of temporal neocortex expression in different groups, and (B) shows the quantification of the data shown in the image in (A). ${ }^{\star} P<0.05$ compared with the untreated control and SB203580 groups; \#P < 0.05 compared with the untreated control and SB203580 groups.

\section{A}

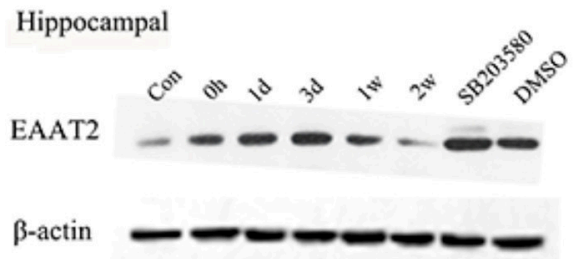

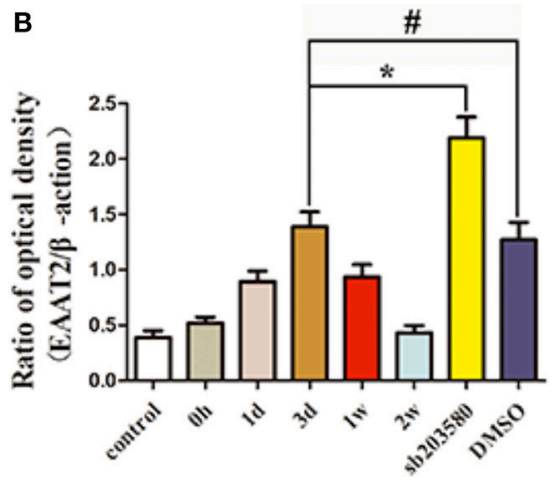

FIGURE 4 | Hippocampal EAAT2 expression in the different groups measured by Western blotting. Panel (A) shows the Western blotting image of hippocampal expression in the different groups, and (B) shows the quantification of the data in the image shown in (A). ${ }^{*} P<0.05$ for the comparison of the SB203580 group with the 3-d group; $\# P>0.05$ for the comparison of the DMSO group with the 3-d group.
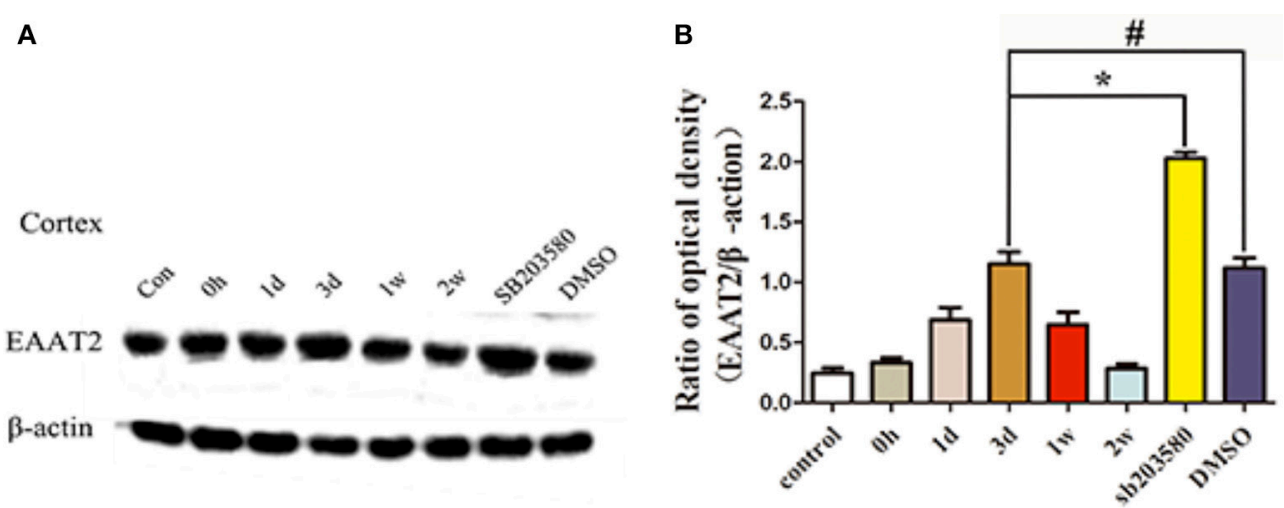

FIGURE 5 | Temporal neocortex EAAT2 expression in the different groups measured by Western blotting. Panel (A) shows the Western blotting image of temporal neocortex expression in the different groups, and (B) shows the quantification of the data in the image shown in (A). ${ }^{*}<0.05$ for the comparison of the SB203580 group with the 3-d group; $\# P<0.05$ for the comparison of the DMSO group with the 3-d group.

EAAT2-positive cells. The number of EAAT2-positive cells in the seizure group at $3 \mathrm{~d}$ was significantly increased $(P<0.05)$ compared with that in the untreated control group. Moreover, the increase in the number of EAAT2-positive cells was significantly greater in the SB203580 group than in the seizure group at $3 \mathrm{~d}(P<0.05)$. 


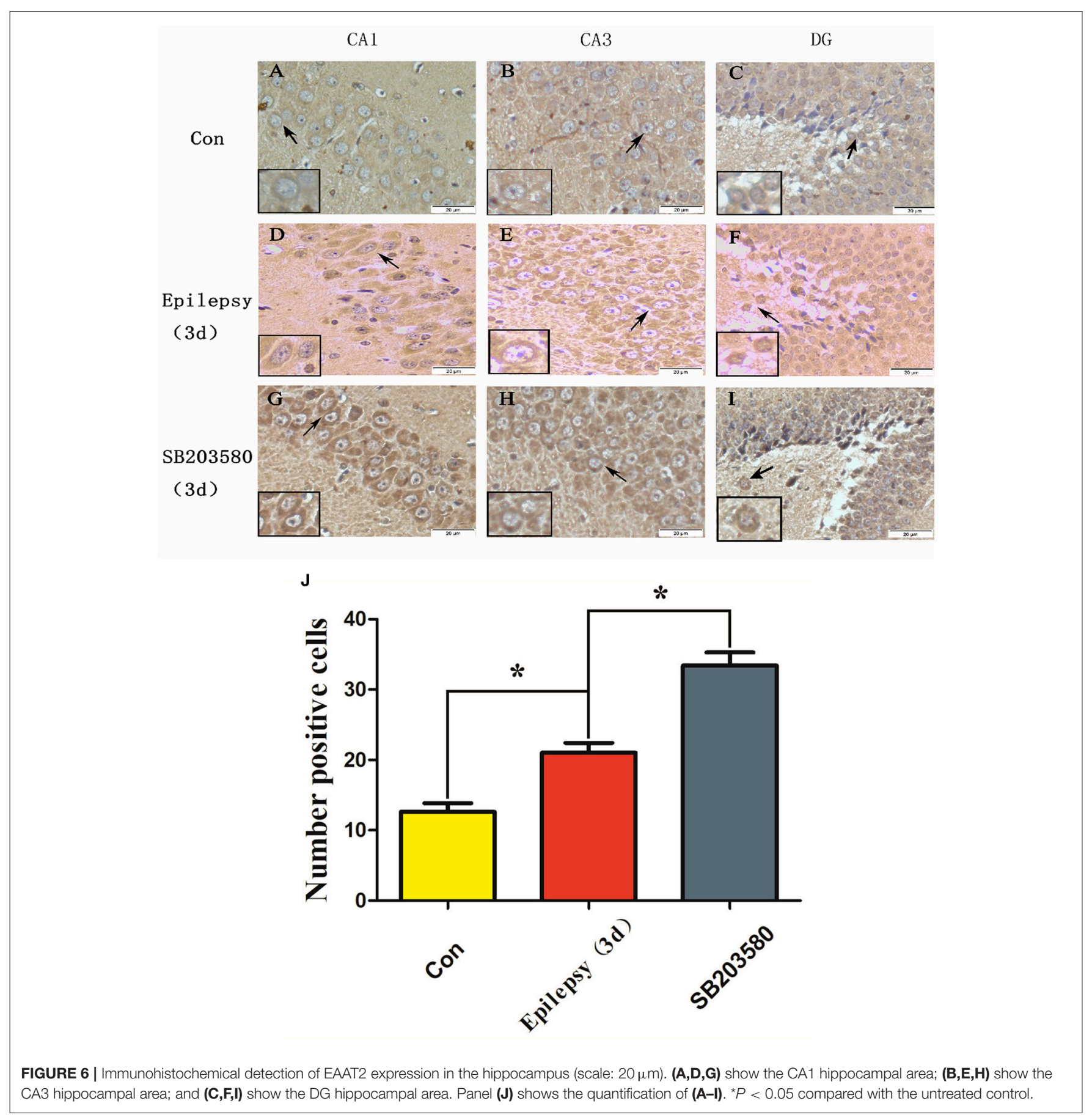

\section{EAAT2 Cellular Localization in the Hippocampus Measured by Immunofluorescence}

Immunohistochemical analyses showed that EAAT2 was expressed mainly in the neuronal cell membrane of the CA1, CA3, and DG hippocampal areas in epileptic rats. To further verify the expression site, we used the doublelabeling immunofluorescence technique to accurately locate the expression of EAAT2 in hippocampal tissue cells. Specimens obtained $3 \mathrm{~d}$ after seizure were used in the analysis. The results showed that EAAT2, the neuronal dendritic specific marker MAP2 and the astroglia-specific marker GFAP were co-expressed in the CA1, CA3, and DG hippocampal areas of rats. In addition, these proteins were expressed mainly in the cytoplasm and membrane of the positively stained cells. Therefore, EAAT2 is expressed in the hippocampus in neuronal cells and is also expressed in some astroglia (Figures 7, 8). 


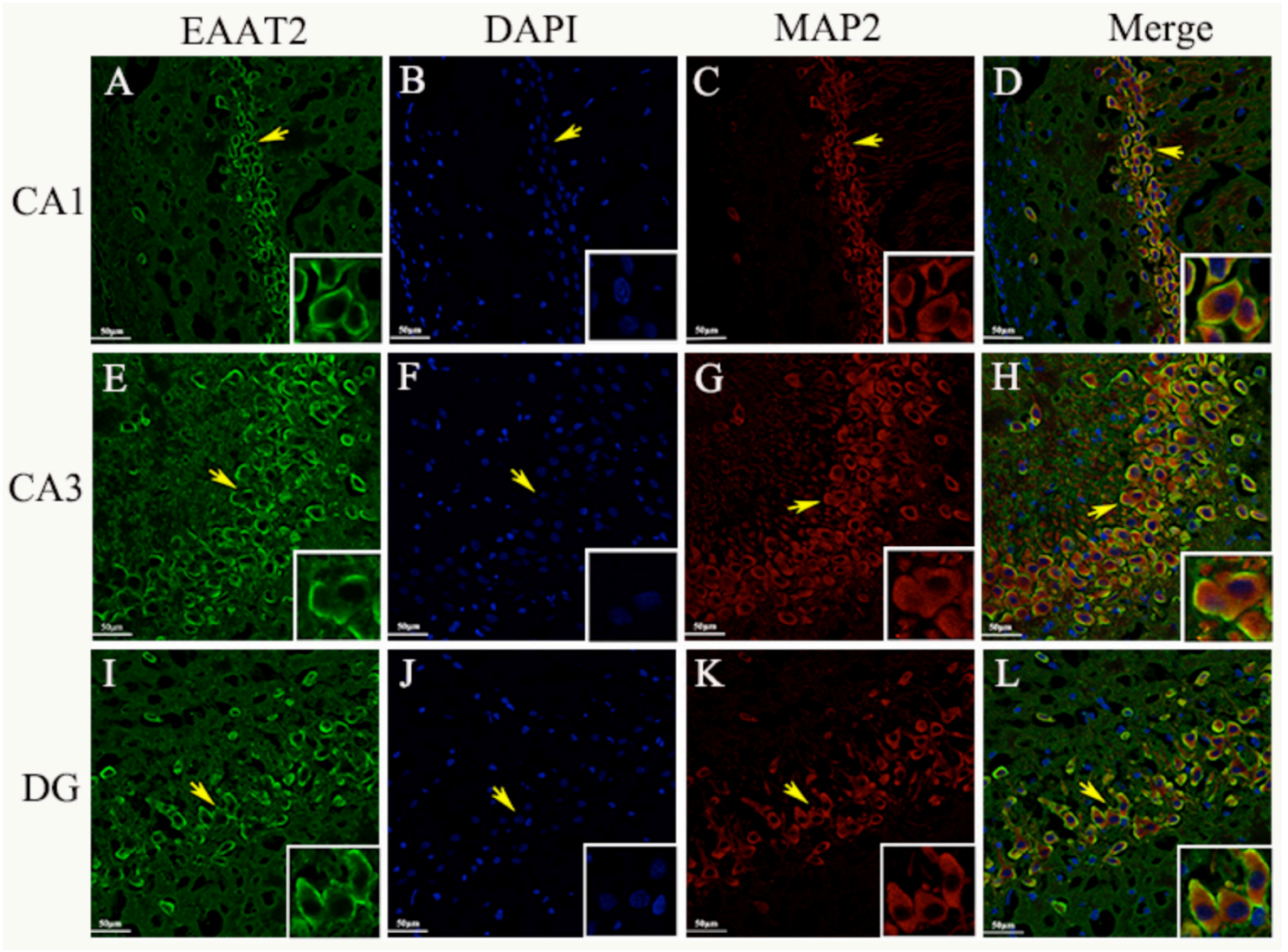

FIGURE 7 | Neuronal cells (A-L). EAAT2 expression in the CA1, CA3, and DG hippocampal areas in rats measured $3 \mathrm{~d}$ after seizure onset by double-labeling immunofluorescence (yellow arrows indicate neurons; white arrows indicate astroglia-like cells) (scale: $20 \mu \mathrm{m}$ ).

\section{DISCUSSION}

Epilepsy is a common chronic disease of the nervous system. Although many antiepileptic drugs are used in clinical settings, the control of seizures with drugs remains difficult in $\sim 30 \%$ of epilepsy patients because the pathogenesis of epilepsy is unclear; thus, there are no effective means for curing epilepsy (20). EAA, particularly via the excitatory effects of Glu, is thought to play a key role in epileptic seizures. Therefore, the study of the EAAT2 glutamate transporter is of great significance.

In this study, we found that EAAT2 expression in the brain tissue of epileptic rats changed dynamically after the onset of epileptic seizures. The EAAT2 expression levels were increased $24 \mathrm{~h}, 3 \mathrm{~d}$, and $1 \mathrm{w}$ after seizure onset but then gradually decreased until returning to the normal levels at $2 \mathrm{w}$. Immunohistochemistry and double-labeling immunofluorescence analyses revealed that EAAT2 was expressed mainly in the cytoplasm and membrane of neurons and astrocytes in the hippocampus of epileptic rats, and these data are consistent with data from previous studies that showed that the EAAT2 glutamate transporter was mainly expressed in the mammalian brain (9). The duration of the first epileptic seizure in rats and their Racine scores were significantly decreased after the administration of SB203580. The expression levels of EAAT2 were also increased, as demonstrated by Western blot and immunohistochemical analyses. We then hypothesized that SB203580 could increase the expression levels of EAAT2 by inhibiting p38 MAPK.

p38 MAPK belongs to the family of stress-activated protein kinases. Stress stimulation (such as ultraviolet light, heat shock, hypertonic environments and protein synthesis inhibitors), cytokines, growth factors, and lipopolysaccharides can activate the p38 MAPK signal transduction pathway $(21,22)$. SE is the strongest source of CNS stimulation and can cause neuronal ATP depletion, shifts in $\mathrm{Ca}^{2+}$, the release of EAA, the production of free radicals and other toxic neuronal reactions, which are effects that lead to the activation of p38 MAPK. ATF-2, NF-AT, and NF- $\mathrm{B}$ can be used as phosphorylation substrates to induce a series of cascade reactions (23). In this study, the expression of T-p38 in brain tissue was significantly downregulated in the SB203580 group compared with the epilepsy group, but no obvious expression of P-p38 was detected in any of the groups. This finding indicates that SB203580 exerts a significant inhibitory effect on p38 MAPK. Therefore, administration of the p38 MAPK inhibitor SB203580 significantly decreased the duration of the first seizure and the Racine scores in rats. These results are consistent with data from previous studies that showed that SB203580 can significantly reduce seizure intensity in a rat 


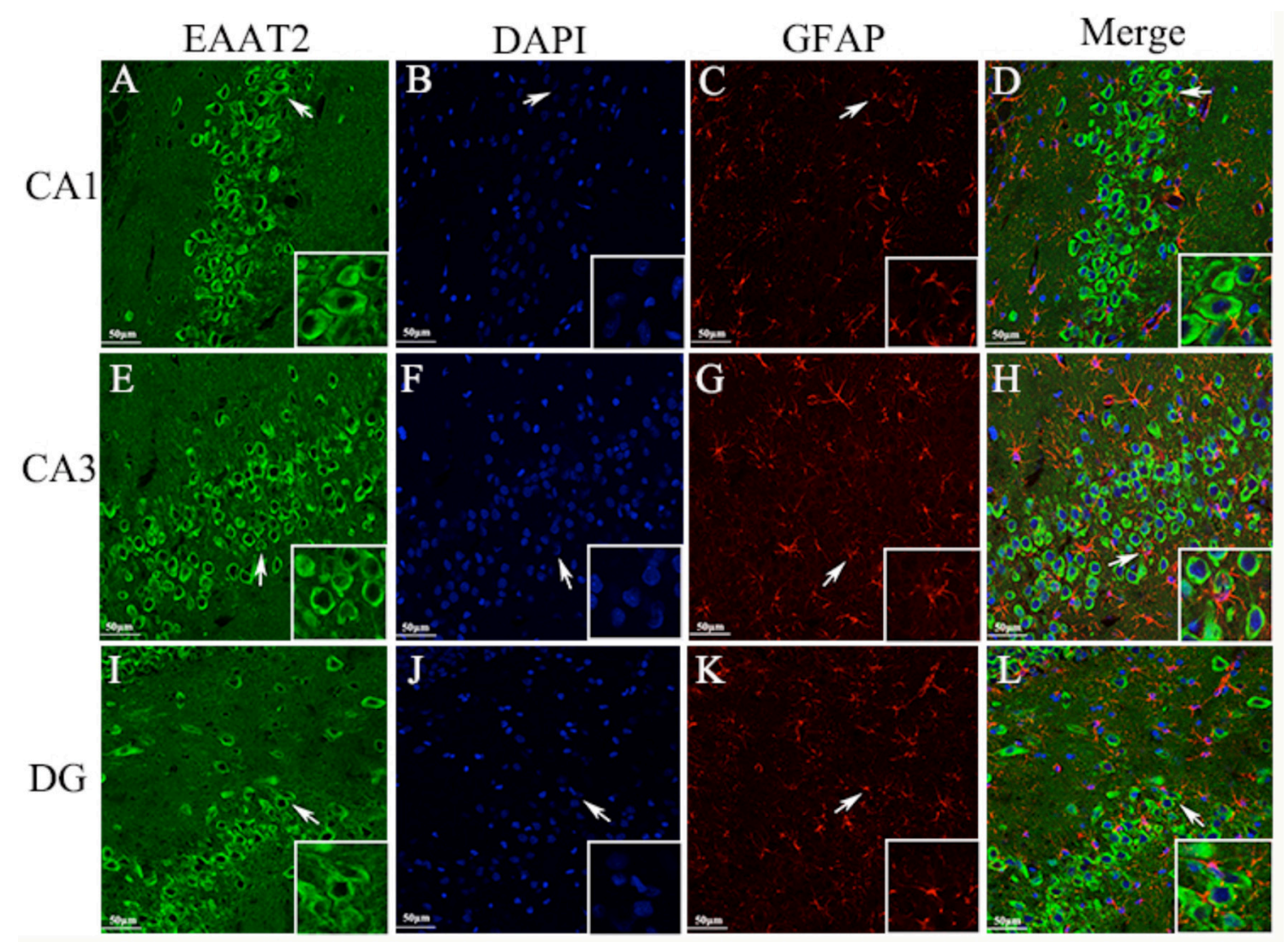

FIGURE 8 | Astroglia (A-L). Same as Figure 7.

model of refractory epilepsy and decrease seizure duration in a rat model of chronic epilepsy $(18,24)$.

After the onset of SE in rats, the glutamate transporter-1 (GLT1) expression levels in the hippocampus began to increase but returned to the baseline levels $30 \mathrm{~d}$ after SE (25). The Western blot results showed that EAAT2 was expressed at basal levels in the control group, but the EAAT2 expression levels were increased after seizure onset, peaking on day 3. These results might be related to the increase in the EAA extracellular concentrations before seizure onset, in the acute seizure stage and at the start of the endogenous protection mechanism. However, with increases in the time after seizure onset $(3 \mathrm{~d}$, $1 \mathrm{w}$, and $2 \mathrm{w}$ ), the expression of EAAT2 was gradually restored to the basal levels. However, after SB203580 administration the EAAT2 expression levels increased, and the seizure duration and Racine scores decreased significantly. These results indicated that SB203580 exerted an antiepileptic effect, which might be related to the increase in EAAT2 expression. Previous studies have shown that presynaptic membranes release Glu through exocytosis. This process increases the synaptic concentration of Glu, and the resulting increased concentration causes $\mathrm{Ca}^{2+}$ and $\mathrm{Na}^{+}$in neurons to flow internally to excessively excite the neurons. The glutamate transporter EAAT2 can rapidly transport synaptic EAAs from the synaptic cleft. As a major transporter that takes up extracellular Glu, EAAT2 causes 80$90 \%$ of these effects (26-30). Thus, EAAT2 plays a very important role in Glu uptake. The increased expression of EAAT2 in brain tissue obtained with SB203580 upregulates Glu uptake, decreases extracellular EAA concentrations and prevents excessive EAA levels from inducing epilepsy. Similarly, increased expression levels of EAAT2 in transgenic mice reduce the number of deaths after SE and reduce neuropathological changes in the hippocampus and seizure progression (31). However, compared with control mice, EAAT2 genetic knockout mice exhibited higher mortality, weight loss and more frequent seizures (32).

In conclusion, EAAT2 plays an important role in epileptic seizures. The expression of EAAT2 can be upregulated by activating transcription or translation (33-36). At the transcriptional level, the EAAT2 promoter can be influenced by most neurochemical signals, such as EGF and TGF- $\alpha$; these signals increase the transcription of EAAT2, whereas TNF- $\alpha$ inhibits the transcription of EAAT2 (37). In addition, Rothstein and others have found that ceftriaxone can increase the protein levels of EAAT2 by activating transcription (38). At the translational level, some small molecule derivatives and drugs can also activate the translation of EAAT2 to increase the expression of EAAT2 (39-41). Kong and others have found that 
the compound LDN/OSU-0212320 can affect the translation of EAAT2, increase the expression of EAAT2 in neurons, and increase the uptake of Glu (31). In this study, administration of the p38 MAPK chemical inhibitor SB203580 also increased the expression levels of EAAT2 in the hippocampus and neocortex of the temporal lobe of rats, but the specific regulatory mechanism is unclear and needs further study.

\section{AUTHOR CONTRIBUTIONS}

ZY completed animal models and thesis writing, ZX guided experiment completion and thesis writing, JW and $\mathrm{HH}$ completed Western blot, PX and CY completed immunohistochemistry, JZ and YP completed immunofluorescence, ZL and JZ completed Statistical analysis.

\section{REFERENCES}

1. Ngugi AK, Bottomley C, Fegan G, Chengo E, Odhiambo R, Bauni $\mathrm{E}$, et al. Premature mortality in active convulsive epilepsy in rural Kenya: causes and associated factors. Neurology (2014) 82:582-9. doi: 10.1212/WNL.0000000000000123

2. Moshe SL, Perucca E, Ryvlin P, Tomson T. Epilepsy: new advances. Lancet (2015) 385:884-98. doi: 10.1016/S0140-6736(14)60456-6

3. During MJ, Spencer DD. Extracellular hippocampal glutamate and spontaneous seizure in the conscious human brain. Lancet (1993) 341:1607-10. doi: 10.1016/0140-6736(93)90754-5

4. Savolainen KM, Loikkanen J, Eerikainen S, Naarala J. Interactions of excitatory neurotransmitters and xenobiotics in excitotoxicity and oxidative stress: glutamate and lead. Toxicol Lett. (1998) 102-103:363-7. doi: 10.1016/S0378-4274(98)00233-1

5. Bradford HF. Glutamate, GABA and epilepsy. Prog Neurobiol. (1995) 47:477-511. doi: 10.1016/0301-0082(95)00030-5

6. Cavus I, Kasoff WS, Cassaday MP, Jacob R, Gueorguieva R, Sherwin RS, et al. Extracellular metabolites in the cortex and hippocampus of epileptic patients. Ann Neurol. (2005) 57:226-35. doi: 10.1002/ana.20380

7. Buckingham SC, Campbell SL, Haas BR, Montana V, Robel S, Ogunrinu T, et al. Glutamate release by primary brain tumors induces epileptic activity. Nat Med. (2011) 17:1269-74. doi: 10.1038/nm.2453

8. Cho CH. New mechanism for glutamate hypothesis in epilepsy. Front Cell Neurosci. (2013) 7:127. doi: 10.3389/fncel.2013.00127

9. Rajkowska G, Miguel-Hidalgo JJ. Gliogenesis and glial pathology in depression. CNS Neurol Disord Drug Targets (2007) 6:219-33. doi: 10.2174/187152707780619326

10. Limpert AS, Cosford ND. Translational enhancers of EAAT2: therapeutic implications for neurodegenerative disease. J Clin Invest. (2014) 124:964-7. doi: 10.1172/JCI74608

11. Fontana AC. Current approaches to enhance glutamate transporter function and expression. J Neurochem. (2015) 134:982-1007. doi: 10.1111/jnc.13200

12. Takahashi K, Foster JB, Lin CL. Glutamate transporter EAAT2: regulation, function, and potential as a therapeutic target for neurological and psychiatric disease. Cell Mol Life Sci. (2015) 72:3489-506. doi: 10.1007/s00018-0151937-8

13. Lee JC, Kumar S, Griswold DE, Underwood DC, Votta BJ, Adams JL. Inhibition of p38 MAPK as a therapeutic strategy. Immunopharmacology (2000) 47:185-201. doi: 10.1016/S0162-3109(00)00206-X

14. Che Y, Yu YM, Han PL, Lee JK. Delayed induction of p38 MAPKs in reactive astrocytes in the brain of mice after KA-induced seizure. Brain Res Mol Brain Res. (2001) 94:157-65. doi: 10.1016/S0169-328X(01)00233-9

15. Haj-Yasein NN, Jensen V, Vindedal GF, Gundersen GA, Klungland A, Ottersen $\mathrm{OP}$, et al. Evidence that compromised $\mathrm{K}+$ spatial buffering contributes to the epileptogenic effect of mutations in the human Kir4.1 gene (KCNJ10). Glia (2011) 59:1635-42. doi: 10.1002/glia.21205

\section{FUNDING}

National Natural Science Foundation of China, 81660227, the study of mitochondria dynamic imbalance regulates the ENT1 in pathogenesis of epilepsy, 2017/01-2020/12 (37万). National Natural Science Foundation of China, 81560224, the study of JNK signal transduction pathway to regulation of ENT1 in rat epileptic discharge and seizures, 2016/01-2019/12 (37万).

\section{ACKNOWLEDGMENTS}

This research was supported by the National Natural Science Foundation of China (No. 81260201, 81271445, and 81201003) and Guizhou Provincial Science and Technology Foundation (No. [2013] 2327).
16. Okamoto OK, Janjoppi L, Bonone FM, Pansani AP, Da Silva AV, Scorza FA, et al. Whole transcriptome analysis of the hippocampus: toward a molecular portrait of epileptogenesis. BMC Genomics (2010) 11:230. doi: 10.1186/1471-2164-11-230

17. Liang J, Chao D, Sandhu HK, Yu Y, Zhang L, Balboni G, et al. $\delta$-Opioid receptors up-regulate excitatory amino acid transporters in mouse astrocytes. Br J Pharmacol. (2014) 171:5417-30. doi: 10.1111/bph.12857

18. Shao $\mathrm{Y}$, Wang $\mathrm{C}$, Hong Z, Chen Y. Inhibition of $\mathrm{p} 38$ mitogen-activated protein kinase signaling reduces multidrug transporter activity and anti-epileptic drug resistance in refractory epileptic rats. J Neurochem. (2016) 136:1096-105. doi: 10.1111/jnc. 13498

19. Bulavin DV, Phillips C, Nannenga B, Timofeev O, Donehower LA, Anderson $\mathrm{CW}$, et al. Inactivation of the Wipl phosphatase inhibits mammary tumorigenesis through p38 MAPK-mediated activation of the p16(Ink4a)p19(Arf) pathway. Nat Genet. (2004) 36:343-50. doi: 10.1038/ng1317

20. Namazi H, Kulish VV, Hussaini J, Hussaini J, Delaviz A, Delaviz F, et al. A signal processing based analysis and prediction of seizure onset in patients with epilepsy. Oncotarget (2016) 7:342-50. doi: 10.18632/oncotarget.6341

21. Brewster JL, De Valoir T, Dwyer ND, Winter E, Gustin MC. An osmosensing signal transduction pathway in yeast. Science (1993) 259:1760-3. doi: 10.1126/science.7681220

22. Souza SC, Palmer HJ, Kang YH, Yamamoto MT, Muliro KV, Paulson KE, et al. TNF-alpha induction of lipolysis is mediated through activation of the extracellular signal related kinase pathway in 3T3-L1 adipocytes. $J$ Cell Biochem. (2003) 89:1077-86. doi: 10.1002/jcb.10565

23. Mizushima H, Zhou CJ, Dohi K, Horai R, Asano M, Iwakura Y, et al. Reduced postischemic apoptosis in the hippocampus of mice deficient in interleukin-1. J Comp Neurol. (2002) 448:203-16. doi: 10.1002/cne.10262

24. Ko AR, Kang TC. Blockade of endothelin B receptor improves the efficacy of levetiracetam in chronic epileptic rats. Seizure (2015) 31:133-40. doi: 10.1016/j.seizure.2015.07.019

25. Hubbard JA, Szu JI, Yonan JM, Binder DK. Regulation of astrocyte glutamate transporter-1 (GLT1) and aquaporin-4 (AQP4) expression in a model of epilepsy. Exp Neurol. (2016) 283:85-96. doi: 10.1016/j.expneurol.2016.05.003

26. Danbolt NC, Storm-Mathisen J, Kanner BI. An $[\mathrm{Na}++$ $\mathrm{K}+$ ]coupled L-glutamate transporter purified from rat brain is located in glial cell processes. Neuroscience (1992) 51:295-310. doi: 10.1016/0306-4522(92)90316-T

27. Haugeto O, Ullensvang K, Levy LM, Chaudhry FA, Honore T, Nielsen M, et al. Brain glutamate transporter proteins form homomultimers. J Biol Chem. (1996) 271:27715-22. doi: 10.1074/jbc.271.44.27715

28. Rothstein JD, Dykes-Hoberg M, Pardo CA, Bristol LA, Jin L, Kuncl RW, et al. Knockout of glutamate transporters reveals a major role for astroglial transport in excitotoxicity and clearance of glutamate. Neuron (1996) 16:675-86. doi: 10.1016/S0896-6273(00)80086-0

29. Tanaka K, Watase K, Manabe T, Yamada K, Watanabe M, Takahashi K, et al. Epilepsy and exacerbation of brain injury in mice lacking 
the glutamate transporter GLT-1. Science (1997) 276:1699-702. doi: 10.1126/science.276.5319.1699

30. Holmseth S, Dehnes Y, Huang YH, Follin-Arbelet VV, Grutle NJ, Mylonakou $\mathrm{MN}$, et al. The density of EAAC1 (EAAT3) glutamate transporters expressed by neurons in the mammalian CNS. J Neurosci. (2012) 32:6000-13. doi: 10.1523/JNEUROSCI.5347-11.2012

31. Kong Q, Takahashi K, Schulte D, Stouffer N, Lin Y, Lin CL. Increased glial glutamate transporter EAAT2 expression reduces epileptogenic processes following pilocarpine-induced status epilepticus. Neurobiol Dis. (2012) 47:145-54. doi: 10.1016/j.nbd.2012.03.032

32. Petr GT, Sun Y, Frederick NM. Conditional deletion of the glutamate transporter GLT-1 reveals that astrocytic GLT-1 protects against fatal epilepsy while neuronal GLT-1 contributes significantly to glutamate uptake into synaptosomes. J Neurosci. (2015) 35:5187-201. doi: 10.1523/JNEUROSCI.4255-14.2015

33. Zelenaia O, Schlag BD, Gochenauer GE, Ganel R, Song W, Beesley JS, et al. Epidermal growth factor receptor agonists increase expression of glutamate transporter GLT-1 in astrocytes through pathways dependent on phosphatidylinositol 3-kinase and transcription factor NF-kappaB. Mol Pharmacol. (2000) 57:667-78. doi: 10.1124/mol.57.4.667

34. Figiel M, Maucher T, Rozyczka J, Bayatti N, Engele J. Regulation of glial glutamate transporter expression by growth factors. Exp Neurol. (2003) 183:124-35. doi: 10.1016/S0014-4886(03)00134-1

35. Tian G, Lai L, Guo H, Lin Y, Butchbach ME, Chang Y, et al. Translational control of glial glutamate transporter EAAT2 expression. J Biol Chem. (2007) 282:1727-37. doi: 10.1074/jbc.M609822200

36. Lee SG, Su ZZ, Emdad L, Gupta P, Sarkar D, Borjabad A, et al. Mechanism of ceftriaxone induction of excitatory amino acid transporter-2 expression and glutamate uptake in primary human astrocytes. J Biol Chem. (2008) 283:13116-23. doi: 10.1074/jbc.M707697200
37. Beart PM, O'shea RD. Transporters for L-glutamate: an update on their molecular pharmacology and pathological involvement. Br J Pharmacol. (2007) 150:5-17. doi: 10.1038/sj.bjp.0706949

38. Rothstein JD, Patel S, Regan MR, Haenggeli C, Huang YH, Bergles DE, et al. Beta-lactam antibiotics offer neuroprotection by increasing glutamate transporter expression. Nature (2005) 433:73-7. doi: 10.1038/nature03180

39. Colton CK, Kong Q, Lai L, Zhu MX, Seyb KI, Cuny GD, et al. Identification of translational activators of glial glutamate transporter EAAT2 through cell-based high-throughput screening: an approach to prevent excitotoxicity. J Biomol Screen (2010) 15:653-62. doi: 10.1177/1087057110 370998

40. Xing X, Chang LC, Kong Q, Colton CK, Lai L, Glicksman MA, et al. Structure-activity relationship study of pyridazine derivatives as glutamate transporter EAAT2 activators. Bioorg Med Chem Lett. (2011) 21:5774-7. doi: 10.1016/j.bmcl.2011.08.009

41. Kong Q, Chang LC, Takahashi K, Liu Q, Schulte DA, Lai L, et al. Smallmolecule activator of glutamate transporter EAAT2 translation provides neuroprotection. J Clin Invest. (2014) 124:1255-67. doi: 10.1172/JCI66163

Conflict of Interest Statement: The authors declare that the research was conducted in the absence of any commercial or financial relationships that could be construed as a potential conflict of interest.

Copyright (๑ 2018 Yang, Wang, Yu, Xu, Zhang, Peng, Luo, Huang, Zeng and Xu. This is an open-access article distributed under the terms of the Creative Commons Attribution License (CC BY). The use, distribution or reproduction in other forums is permitted, provided the original author(s) and the copyright owner(s) are credited and that the original publication in this journal is cited, in accordance with accepted academic practice. No use, distribution or reproduction is permitted which does not comply with these terms. 